\title{
Microalbuminuria, macroalbuminuria and uncontrolled blood pressure among diagnosed hypertensive patients: the aspect of racial disparity in the NHANES study
}

\begin{abstract}
Xuefeng Liu ${ }^{1,2}$, Kesheng Wang ${ }^{1,2}$, Liang Wang ${ }^{1}$ and Dennis Tsilimingras ${ }^{3}$
Accumulating evidence reveals that albuminuria may exacerbate uncontrolled blood pressure (BP) in hypertensive patients. However, racial differences in the associations of albuminuria with uncontrolled BP among diagnosed hypertensives have not been evaluated. A total of 6147 diagnosed hypertensive subjects aged $\geqslant 18$ years were collected from the National Health and Nutrition Examination Survey 1999-2008 with stratified multistage sampling designs. Odds ratios (ORs), relative ORs and 95\% confidence intervals (Cls) in uncontrolled BP, and the different effects of microalbuminuria and macroalbuminuria on continuous BP were estimated using weighted logistic models and linear regression models. Hypertensive subjects with microalbuminuria and macroalbuminuria were more likely to have uncontrolled BP and higher average systolic BP (SBP) in all individual racial groups. Microalbuminuria was associated with isolated uncontrolled SBP in non-Hispanic blacks and whites, and macroalbuminuria was associated with isolated uncontrolled SBP and diastolic BP (DBP) and high average DBP only in non-Hispanic blacks. Compared with non-Hispanic whites, non-Hispanic blacks and Mexicans had lower associations of microalbuminuria with uncontrolled $\mathrm{BP}$ (relative $\mathrm{OR}=0.68,95 \% \mathrm{Cl}=0.48-0.97$ for blacks vs whites; relative $\mathrm{OR}=0.62,95 \%$ $\mathrm{Cl}=0.42-0.93$ for Mexicans vs. whites) and isolated uncontrolled $\mathrm{SBP}$ (relative $\mathrm{OR}=0.62,95 \% \mathrm{Cl}=0.43-0.90$ for blacks vs. whites; relative $\mathrm{OR}=0.45,95 \% \mathrm{Cl}=0.29-0.71$ for Mexicans vs. whites). The association of microalbuminuria with uncontrolled BP was lower in non-Hispanic blacks and Mexicans than in non-Hispanic whites. Health providers need to improve care for mildly elevated albumin excretion rates in non-Hispanic white hypertensive patients while maintaining the quality of care in non-Hispanic blacks and Mexicans.
\end{abstract}

Hypertension Research (2013) 36, 1100-1106; doi:10.1038/hr.2013.87; published online 15 August 2013

Keywords: albuminuria; isolated uncontrolled diastolic BP; isolated uncontrolled systolic BP; racial disparity; uncontrolled BP

\section{INTRODUCTION}

Hypertension or high blood pressure (BP) has been known as a major risk factor associated with cardiovascular events and renal diseases. ${ }^{1-5}$ The goal of antihypertensive therapy is to lower elevated BP below $140 / 90 \mathrm{~mm} \mathrm{Hg}$ to reduce the risk of cardiovascular disease and slow down the progression of renal disease. ${ }^{6}$ One hurdle to controlling high BP is the racial disparity in cardiovascular risk factors related to uncontrolled hypertension. Much of the racial disparity in cardiovascular outcomes can be attributed to the excess burden of uncontrolled BP in specific racial populations. Albuminuria has been linked to uncontrolled BP in subjects with hypertension or chronic kidney disease. ${ }^{7-9}$ Although many studies showed racial differences in the prevalence of albuminuria and uncontrolled hypertension, ${ }^{10-13}$ information on racial differences in the relations between albuminuria and uncontrolled BP among hypertensive patients has not been reported. Examining racial differences in the association of albuminuria with uncontrolled BP may contribute to a better understanding of the role of albuminuria in racial differences in uncontrolled BP. This examination may also lead to the better attainment of goal BP as specified by the Seventh Report of the Joint National Committee on Prevention, Detection, Evaluation and Treatment of High Blood Pressure ${ }^{14}$ among hypertensive patients in specific racial populations.

In this study, we evaluated racial differences in the association of albuminuria with uncontrolled BP in non-Hispanic blacks, Mexicans and non-Hispanic whites with diagnosed hypertension, using the continuous National Health and Nutrition Examination Survey (NHANES) data from the period 1999-2008. Albuminuria was

${ }^{1}$ Department of Biostatistics and Epidemiology, East Tennessee State University, Johnson City, TN, USA; ${ }^{2}$ nstitute for Quantitative Biology, East Tennessee State University, Johnson City, TN, USA and ${ }^{3}$ Center on Patient Safety, Division of Health Affairs, Department of Family Medicine and Rural Health, College of Medicine at the Florida State University, Tallahassee, FL, USA

Correspondence: Professor X Liu, Department of Biostatistics and Epidemiology, East Tennessee State University, 46D Lamb Hall, Johnson City, TN 37614 , USA. E-mail: lix01@etsu.edu

Received 7 February 2013; revised 17 April 2013; accepted 9 May 2013; published online 15 August 2013 
reported to include microalbuminuria and macroalbuminuria defined by the ratio of spot urine albumin and creatinine. Our goal was to identify racial disparities in the relationships among microalbuminuria, macroalbuminuria and uncontrolled BP outcomes, which would allow targeting of these relationships in an effort to effectively control high BP and reduce race-specific cardiovascular diseaserelated morbidities and mortality among diagnosed hypertensive patients in different racial populations.

\section{METHODS}

\section{Study design and participants}

The NHANES was conducted by the National Center for Health Statistics of the Centers for Disease Control and Prevention. It was a survey program designed to monitor the health and nutrition status of adults and children in the non-institutionalized population in the US. All of the participants gave informed consent, and the study received approval from the Centers for Disease Control and Prevention Institutional Review Board. The NHANES survey consisted of interviews and examinations. Interviews were performed in the participants' home to obtain information regarding socio-demographic characteristics, and the examinations were conducted in a mobile examination center to obtain measurements of physical and clinical/laboratory factors. The continuous NHANES program began in 1999 and proceeded in a 2-year cycle. It was a stratified multistage survey with complex probability sampling designs. The detailed measurement procedures and protocols have been described in a previous publication ${ }^{15}$ and can also be found on the NHANES web site (http:// www.cdc.gov/nchs/about/major/nhanes/datalink.htm).

Our study sample was built on the basis of the continuous NHANES program 1999-2008. Participants younger than 18 years were excluded from the study. Other participants who were excluded from the study included individuals who were interviewed but not examined, pregnant women, individuals who were not diagnosed as hypertensive patients and patients who were identified as non-Mexican Hispanic or multiracial. In NHANES from 1999-2008, there were a total of 12825 non-Hispanic whites, 5782 nonHispanic blacks and 5986 Mexicans aged 18 years and older who were both interviewed and examined. After excluding 1127 pregnant women, 23466 participants were left. Among them, there were a total of 6147 participants with diagnosed hypertension included in the present study.

\section{BP outcomes}

Systolic BP (SBP) and diastolic BP (DBP) were manually measured up to four times by a trained examiner using a mercury sphygmomanometer according to a standard protocol. ${ }^{16}$ The final SBP and DBP were calculated as the average of the last two measurements of SBP or DBP for participants who had three or four measurements, whereas the last measurement was used for participants with only two measurements, and the single measurement was used for participants from whom only one measurement was taken. Participants were defined to have diagnosed hypertension if they self-reported hypertension diagnosed by a doctor and had BP measurements $\geqslant 140 / 90 \mathrm{~mm} \mathrm{Hg}$ or if they were taking antihypertensive medications. Among participants with diagnosed hypertension, uncontrolled BP was defined as a BP level of $\geqslant 140 / 90 \mathrm{~mm} \mathrm{Hg}$ in nondiabetic persons and $\geqslant 130 / 80 \mathrm{mmHg}$ in diabetic persons; isolated uncontrolled SBP was defined as SBP levels $\geqslant 140 \mathrm{~mm} \mathrm{Hg}$ and DBP levels $<90 \mathrm{~mm} \mathrm{Hg}$ in non-diabetics or as SBP levels $\geqslant 130 \mathrm{~mm} \mathrm{Hg}$ and DBP levels $<80 \mathrm{~mm} \mathrm{Hg}$ in diabetics; and isolated uncontrolled DBP was defined as DBP levels $\geqslant 90 \mathrm{~mm} \mathrm{Hg}$ and SBP levels $<140 \mathrm{~mm} \mathrm{Hg}$ in non-diabetics or as DBP levels $\geqslant 80 \mathrm{~mm} \mathrm{Hg}$ and SBP levels $<130 \mathrm{~mm} \mathrm{Hg}$ in diabetics in accordance with the Seventh Report of the Joint National Committee on Prevention, Detection, Evaluation and Treatment of High Blood Pressure. ${ }^{14}$

\section{Primary factors: microalbuminuria and macroalbuminuria}

Albuminuria is a condition of extra albumin excretion occurring in human urine. The level of albuminuria was determined by the ratio of spot urine albumin and creatinine. Participants were defined to have microalbuminuria if the level of the urinary albumin to creatinine ratio (UACR) was $\geqslant 30 \mathrm{mg} \mathrm{g}^{-1}$ and $<300 \mathrm{mg} \mathrm{g}^{-1}$, and to have macroalbuminuria if the level of UACR was $\geqslant 300 \mathrm{mgg}^{-1} \cdot{ }^{17}$ Urinary albumin was measured using a solid-phase fluorescent immunoassay with a digital Sequoia-Turner model 450 fluorometer (Sequoia-Turner Corp, Mountain View, CA, USA). Urinary creatinine was measured colorimetrically using a Jaffé rate reaction on a Beckman Synchron AS/ASTRA clinical analyzer (Beckman Instruments, Brea, CA, USA)

\section{Other characteristics}

Information on age, gender, race/ethnicity and education was obtained from self-reported questionnaires. Participants were defined as poor if the family's poverty index ratio $<1.0$. The poverty index ratio is the ratio of the family's total income to the family's appropriate poverty threshold (US Census Bureau, 2003, 2007). A history of smoking was obtained from household interviews for participants aged $\geqslant 20$ years and from the examination center for participants aged $12-19$ years. Smokers were defined as subjects aged $\geqslant 20$ years who smoked $\geqslant 100$ cigarettes in their lives or subjects aged 18 to 19 years who had ever tried smoking a whole cigarette. Current smokers included smokers aged $\geqslant 20$ years who reported current smoking and smokers aged 18 to 19 years who reported smoking in the past month; former smokers included smokers aged $\geqslant 20$ years who did not report currently smoking and those smokers aged 18 to 19 years who did not report smoking in the past month. Body mass index was calculated as weight in kilograms divided by the square of height in meters. Pregnancy was determined in women by a self-reported questionnaire and a urine pregnancy test. Serum cholesterol was measured enzymatically by the generation of hydrogen peroxide. High cholesterol was defined based on the concentrations of serum cholesterol in the blood sample. A participant was considered to have high cholesterol if his or her serum cholesterol was $\geqslant 200 \mathrm{mg} \mathrm{dl}^{-1}$. Diabetes was defined by a positive response to any of the questions, 'Have you ever been told by a doctor that you have diabetes?'; 'Are you now taking insulin?'; 'Are you now taking diabetes pills to lower your blood sugar?' The definition did not include patients with only a fasting plasma glucose of $126 \mathrm{mg} \mathrm{dl}^{-1}$ or higher (that is, undiagnosed diabetes).

\section{Statistical analysis}

The characteristics of the participants with diagnosed hypertension were evaluated and compared between non-Hispanic blacks, Mexicans and nonHispanic whites using $\chi^{2}$ statistics for categorical variables and Wald tests for continuous variables. BP control rates and the prevalence of the risk factors of interest were age-adjusted by direct standardization to the NHANES 19992008 population with diagnosed hypertension. Stratum, cluster and weight design techniques for survey data were considered to incorporate sampling weights into data analysis to generalize the results to the whole population of non-institutionalized, diagnosed hypertensive patients in the US

To examine racial differences in the associations of microalbuminuria and macroalbuminuria with uncontrolled BP and continuous $\mathrm{BP}$, we conducted multiple logistic regression models to evaluate the associations of primary factors with uncontrolled BP and multiple linear regression models to evaluate the associations of primary factors with continuous BP in each racial population as well as in the combined population. After adjusting for the confounding influence from each subject's age, gender, education, family poverty index ratio, smoking status, body mass index, serum cholesterol, diabetes status and prescribed medications used for hypertension, the adjusted odds ratios (ORs) and 95\% confidence intervals (CIs) of uncontrolled BP, SBP and DBP were obtained from multiple logistic regression models to examine their relations with microalbuminuria and macroalbuminuria. Regression coefficients of the above factors on continuous SBP and DBP were obtained from multiple linear regression models to examine how continuous SBP and DBP change with them in each racial group.

To test the significance of racial differences in the associations of microalbuminuria and macroalbuminuria with uncontrolled BP and continuous BP among diagnosed hypertensive patients, relative ORs and 95\% CIs of uncontrolled BP, SBP and DBP (categorical end points) and the effect differences in continuous SBP and DBP associated with microalbuminuria and macroalbuminuria were derived from weighted multiple logistic regression models and weighted multiple linear regression models, respectively. This was attained by adding race, albuminuria (micro and macro) and the interaction of 
race and albuminuria into the models. The relative ORs were calculated as the ORs of uncontrolled BP, SBP and DBP for microalbuminuria and macroalbuminuria in one racial group divided by the corresponding ORs of uncontrolled BP, SBP and DBP in another racial group. The effect differences were derived as the differences in effects of the primary factors on continuous SBP and DBP between racial groups. The models were adjusted for the same group of confounding factors as were used previously. All data analyses were completed using SAS version 9.2 (SAS Institute, Cary, NC, USA).

\section{RESULTS}

The average age of participants with diagnosed hypertension in the study population was $60.48 \pm 0.33$ years. A total of $55.12 \pm 0.87 \%$ of the participants were female; $15.82 \pm 1.37 \%$ were non-Hispanic blacks, $4.01 \pm 0.58 \%$ were Mexicans and $80.17 \pm 1.55 \%$ were non-Hispanic whites. After adjustment for age, the obesity rate was $46.21 \pm 0.72 \%$. The prevalence of high cholesterol, diabetes and albuminuria (albumin-creatinine ratio levels $\geqslant 30 \mathrm{mgg}^{-1}$ ) were $49.66 \pm 0.97 \%, 24.46 \pm 0.78 \%$ and $20.41 \pm 0.52 \%$, respectively The overall rate of uncontrolled BP among the participants with diagnosed hypertension in NHANES $1999-2008$ was $50.83 \pm 0.95 \%$.

Among the diagnosed hypertensive patients in the study, nonHispanic whites were older and had higher rates of being former smokers than non-Hispanic blacks and Mexicans. There were more females than males with diagnosed hypertension in each of the racial groups, especially for non-Hispanic blacks. Overall, Non-Hispanic blacks and Mexicans were poorer, received less education and had greater mean body mass index and higher obesity rates than nonHispanic whites. The prevalence of diabetes, microalbuminuria and macroalbuminuria were highest in Mexicans and lowest in nonHispanic whites. Compared with non-Hispanic whites, non-Hispanic blacks and Mexicans had a higher mean SBP and a higher rate of uncontrolled BP, and non-Hispanic blacks had a higher mean DBP (Table 1).

Table 1 Age-adjusted characteristics of subjects with diagnosed hypertension by race in NHANES 1999-2008

\begin{tabular}{|c|c|c|c|}
\hline \multirow[b]{2}{*}{ Characteristics } & \multicolumn{3}{|c|}{ Means or percentages (95\% confidence intervals) } \\
\hline & Non-Hispanic blacks & Mexicans & Non-Hispanic whites \\
\hline Count & 1772 & 955 & 3420 \\
\hline Age (years) & $56.30(55.37,57.23)$ & $56.40(55.03,57.78)$ & $61.51(60.70,62.33)^{\ddagger}$ \\
\hline \multicolumn{4}{|l|}{ Gender } \\
\hline Female (\%) & $63.24(60.86,65.61)$ & $56.00(52.78,59.24)$ & $54.77(52.98,56.56)^{\ddagger}$ \\
\hline \multicolumn{4}{|l|}{ Education } \\
\hline High school or below (\%) & $66.19(62.68,69.69)$ & $78.44(75.37,81.51)$ & $51.84(48.43,55.24)^{\ddagger}$ \\
\hline \multicolumn{4}{|l|}{ Family poverty income ratio } \\
\hline Mean & $2.28(2.15,2.42)$ & $2.08(1.88,2.29)$ & $3.03(2.93,3.14)^{\ddagger}$ \\
\hline Poverty (\%) & $23.07(19.49,26.66)$ & $27.52(22.08,32.96)$ & $8.29(6.79,9.78)^{\ddagger}$ \\
\hline \multicolumn{4}{|l|}{ Smoking } \\
\hline Former smoking (\%) & $29.50(27.29,31.70)$ & $31.63(28.24,35.03)$ & $39.74(37.92,41.56)^{\ddagger}$ \\
\hline Current smoking (\%) & $24.82(22.29,27.36)$ & $17.19(15.23,19.15)$ & $19.20(17.47,20.92)$ \\
\hline \multicolumn{4}{|l|}{ Body mass index } \\
\hline Mean $\left(\mathrm{kg} \mathrm{m}^{-2}\right)$ & $31.71(31.36,32.06)$ & $30.73(30.33,31.13)$ & $30.40(30.14,30.67)^{\ddagger}$ \\
\hline Obesity (\%) & $53.32(50.67,55.97)$ & $48.77(45.25,52.29)$ & $45.00(43.39,46.60)^{\dagger}$ \\
\hline \multicolumn{4}{|l|}{ Serum cholesterol } \\
\hline Mean (mg dl ${ }^{-1}$ ) & $203.15(199.70,206,60)$ & $200.63(197.39,203.86)$ & $202.28(200.30,204.26)$ \\
\hline High cholesterol (\%) & $50.16(46.76,53.56)$ & $49.04(45.61,52.47)$ & $49.73(47.43,52.04)$ \\
\hline Currently taking medications for hypertension (\%) & $95.18(94.33,96.04)$ & $92.65(90.92,94.39)$ & $94.61(93.75,95.48)$ \\
\hline Diabetes (\%) & $37.99(35.24,40.74)$ & $41.60(38.33,44.88)$ & $21.47(19.66,23.29)^{\ddagger}$ \\
\hline \multicolumn{4}{|l|}{ Albuminuria } \\
\hline Microalbuminuria (\%) & $20.12(18.04,22.19)$ & $23.21(20.01,26.41)$ & $14.92(13.83,16.01)^{\ddagger}$ \\
\hline Macroalbuminuria (\%) & $7.42(5.91,8.93)$ & $7.88(5.95,9.81)$ & $3.59(2.88,4.31)^{\ddagger}$ \\
\hline \multicolumn{4}{|l|}{ Blood pressure } \\
\hline Systolic BP (mm Hg) & $141.04(139.72,142.37)$ & $141.51(139.42,143.61)$ & $136.43(135.62,137.24)^{\ddagger}$ \\
\hline Diastolic BP (mm Hg) & $73.17(72.19,74.15)$ & $71.22(69.87,72.56)$ & $70.50(69.89,71.12)^{\ddagger}$ \\
\hline Uncontrolled BP (\%) & $57.84(55.38,60.31)$ & $59.38(55.02,63.74)$ & $49.03(46.77,51.29)^{\ddagger}$ \\
\hline Isolated uncontrolled SBP (\%) & $45.45(42.72,48.17)$ & $49.70(45.32,54.08)$ & $41.29(39.01,43.56)$ \\
\hline Isolated uncontrolled DBP (\%) & $7.57(6.02,9.11)$ & $7.59(4.94,10.24)$ & $6.00(4.86,7.13)$ \\
\hline
\end{tabular}

Abbreviations: BP, blood pressure; systolic blood pressure; DBP, NHANES, National Health and Nutrition Examination Survey; SBP, diastolic blood pressure. Notes: data were age adjusted by direct standardization to the diagnosed hypertensive population in NHANES 1999-2008 except for age-specific estimates. $\ddagger P<0.001, \dagger P<0.01$, for overall differences of means or percentages of characteristics across racial groups. 
Table 2 Adjusted odds ratios of uncontrolled blood pressure and regression coefficients on continuous blood pressure for albuminuria by race in subjects with diagnosed hypertension in NHANES 1999-2008

\begin{tabular}{|c|c|c|c|c|}
\hline & \multicolumn{4}{|c|}{ Regression coefficients or odds ratios (95\% confidence intervals) } \\
\hline & Non-Hispanic blacks & Mexicans & Non-Hispanic whites & All \\
\hline \multicolumn{5}{|c|}{ Uncontrolled BP (Yes/No) } \\
\hline Microalbuminuria & $1.69(1.23,2.32)^{\dagger}$ & $1.77(1.17,2.67)^{\dagger}$ & $2.45(1.92,3.12)^{\ddagger}$ & $2.28(1.87,2.78)^{\ddagger}$ \\
\hline Macroalbuminuria & $3.70(2.02,6.76)^{\ddagger}$ & $3.06(1.20,7.83)^{*}$ & $1.88(1.01,3.52)^{*}$ & $2.34(1.48,3.72)^{\ddagger}$ \\
\hline \multicolumn{5}{|c|}{ Isolated uncontrolled SBP (Yes/No) } \\
\hline Microalbuminuria & $1.49(1.08,2.06)^{*}$ & $1.36(0.83,2.22)$ & $2.42(1.89,3.12)^{\ddagger}$ & $2.23(1.81,2.74)^{\ddagger}$ \\
\hline Macroalbuminuria & $2.84(1.54,5.23)^{\ddagger}$ & $1.99(0.70,5.68)$ & $1.81(0.88,3.73)$ & $2.00(1.15,3.47)^{*}$ \\
\hline \multicolumn{5}{|c|}{ Isolated uncontrolled DBP (Yes/No) } \\
\hline Microalbuminuria & $1.61(0.65,4.00)$ & $2.49(0.69,8.92)$ & $1.80(0.65,4.97)$ & $1.71(0.79,3.69)$ \\
\hline Macroalbuminuria & $6.84(2.23,20.94)^{\ddagger}$ & $2.62(0.25,27.52)$ & NA & $1.48(0.56,3.92)$ \\
\hline \multicolumn{5}{|c|}{ Continuous SBP $(\mathrm{mm} \mathrm{Hg})$} \\
\hline Microalbuminuria & $7.16(3.27,11.04)^{\ddagger}$ & $6.79(1.89,11.70)^{\dagger}$ & $8.53(6.55,10.51)^{\ddagger}$ & $8.37(6.63,10.11)^{\ddagger}$ \\
\hline Macroalbuminuria & $13.83(8.21,19.45)^{\ddagger}$ & $15.28(4.76,25.79)^{\dagger}$ & $14.56(6.00,23.12)^{\ddagger}$ & $14.83(8.83,20.84)^{\ddagger}$ \\
\hline \multicolumn{5}{|c|}{ Continuous DBP ( $\mathrm{mm} \mathrm{Hg})$} \\
\hline Microalbuminuria & $2.27(-0.45,4.99)$ & $2.22(-1.67,6.11)$ & $1.86(0.08,3.64)^{*}$ & $1.95(0.45,3.95)^{*}$ \\
\hline Macroalbuminuria & $5.74(2.28,9.21)^{\dagger}$ & $4.65(-1.77,11.07)$ & $-0.56(-4.84,3.73)$ & $1.43(-1.71,4.57)$ \\
\hline
\end{tabular}

After adjustment for each subject's age, gender, education, family poverty index ratio, smoking status, body mass index, serum cholesterol, diabetes status and prescribed medications used for hypertension, microalbuminuria and macroalbuminuria were more likely to be associated with uncontrolled BP and uncontrolled SBP in the overall population of patients diagnosed with hypertension (last column in Table 2). Separate analyses showed that compared with normoalbuminuria, participants with microalbuminuria had a $69 \%$ $(\mathrm{OR}=1.69,95 \% \mathrm{CI}=1.23-2.32), 77 \%(\mathrm{OR}=1.77,95 \% \mathrm{CI}=1.17-$ $2.67)$ and a $145 \%(\mathrm{OR}=2.45,95 \% \mathrm{CI}=1.92-3.12)$, and participants with macroalbuminuria had a $270 \%(\mathrm{OR}=3.70,95 \% \mathrm{CI}=2.02-$ $6.76), 206 \%(\mathrm{OR}=3.06,95 \% \mathrm{CI}=1.20-7.83)$ and a $88 \%(\mathrm{OR}=1.88$, $95 \% \mathrm{CI}=1.01-3.52)$ higher chance of having uncontrolled BP in non-Hispanic blacks, Mexicans and non-Hispanic whites, respectively. Moreover, microalbuminuria was associated with isolated uncontrolled SBP in non-Hispanic blacks and whites, but not in Mexicans; macroalbuminuria was associated with uncontrolled SBP and DBP only in non-Hispanic blacks (Table 2).

Among the participants with diagnosed hypertension, the average SBP increased by 8.37 and $14.83 \mathrm{~mm} \mathrm{Hg}$ for microalbuminuria and macroalbuminuria, respectively, and the average DBP increased by $1.95 \mathrm{~mm} \mathrm{Hg}$ for microalbuminuria (Table 2). Separate analyses revealed that participants identified as non-Hispanic blacks, Mexicans and non-Hispanic whites with microalbuminuria had an average SBP that was $7.16(95 \% \mathrm{CI}=3.27-11.04), 6.79(95 \% \mathrm{CI}=1.89-11.70)$ and $8.53(95 \% \mathrm{CI}=6.55-10.51) \mathrm{mm} \mathrm{Hg}$ higher, respectively, than participants with normoalbuminuria, whereas those with macroalbuminuria had an average SBP that was $13.83(95 \% \mathrm{CI}=8.21-19.45)$, $15.28(95 \% \mathrm{CI}=4.76-25.79)$ and $14.56(95 \% \mathrm{CI}=6.00-23.12) \mathrm{mm}$ $\mathrm{Hg}$ higher, respectively, than participants with normoalbuminuria.
The average DBP increased by $1.86(95 \% \mathrm{CI}=0.08-3.64) \mathrm{mm} \mathrm{Hg}$ for microalbuminuria in non-Hispanic whites and increased by 5.74 (95\% CI $=2.28-9.21) ~ m m ~ H g$ for macroalbuminuria in non-Hispanic blacks.

The relative OR of uncontrolled BP, SBP and DBP and the effect differences in continuous SBP and DBP were calculated to compare the associations of microalbuminuria and macroalbuminuria with these BP end points across racial groups (see Table 3). From Table 3, we can see that the associations of microalbuminuria with uncontrolled BP were lower in non-Hispanic blacks and Mexicans than in non-Hispanic whites (non-Hispanic blacks vs non-Hispanic whites: relative $\mathrm{OR}=0.68,95 \% \mathrm{CI}=0.48-0.97$; Mexicans $v s$. non-Hispanic whites: relative $\mathrm{OR}=0.62,95 \% \mathrm{CI}=0.42-0.93)$. Similar significant results were found with isolated uncontrolled SBP: (non-Hispanic blacks $v$ s. non-Hispanic whites: relative $\mathrm{OR}=0.62,95 \% \mathrm{CI}=0.43-$ 0.90; Mexicans vs. non-Hispanic whites: relative $\mathrm{OR}=0.45,95 \%$ $\mathrm{CI}=0.29-0.71)$.

\section{DISCUSSION}

This study reports the racial differences in the associations of microalbuminuria and macroalbuminuria with uncontrolled BP and continuous BP among participants with diagnosed hypertension in NHANES 1999-2008. Microalbuminuria and macroalbuminuria were found to be associated with uncontrolled BP, isolated uncontrolled SBP and continuous SBP in the overall population. Separate analyses showed that microalbuminuria and macroalbuminuria were significantly associated with uncontrolled BP and continuous SBP in each separate population of non-Hispanic blacks, Mexicans and nonHispanic whites. 
Table 3 Relative odds ratios of uncontrolled blood pressure and effect differences in continuous blood pressure for albuminuria between racial groups of subjects with diagnosed hypertension in NHANES 1999-2008

Relative odds ratios or effect differences (95\% confidence intervals)

\section{Uncontrolled BP (Yes/No) \\ Microalbuminuria \\ Macroalbuminuria}

Isolated uncontrolled SBP (Yes/No)

Microalbuminuria

Macroalbuminuria

Isolated uncontrolled DBP (Yes/No)

Microalbuminuria

Macroalbuminuria

Continuous SBP $(\mathrm{mm} \mathrm{Hg})$

Microalbuminuria

Macroalbuminuria

\section{Continuous DBP $(\mathrm{mm} \mathrm{Hg})$}

Microalbuminuria

Macroalbuminuria
$0.68(0.48,0.97)^{*}$

$1.57(0.62,3.98)$

$0.62(0.43,0.90)^{*}$

$1.48(0.57,3.89)$

$0.59(0.13,2.68)$

NA

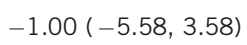

$-1.00(-5.58,3.58)$

$-2.83(-12.75,7.08)$
$0.62(0.42,0.93)^{*}$

$0.87(0.28,2.70)$

$0.45(0.29,0.71)^{\ddagger}$

$0.61(0.20,1.87)$

$1.33(0.36,5.01)$

NA

$-3.77(-7.99,0.45)$
$-5.19(-18.45,8.07)$

$-0.05(-3.82,3.73)$

$2.27(-4.43,8.98)$
$1.09(0.67,1.79)$

$1.81(0.58,5.67)$

$1.38(0.83,2.28)$

$2.43(0.85,6.93)$

$0.44(0.08,2.31)$

$3.16(0.37,27.32)$

$2.77(-2.96,8.49)$

$2.36(-9.14,13.85)$

minuria $\quad 5.67(-0.02,11.35)$

$0.46(-2.71,3.63)$

$0.50(-4.51,5.52)$

$3.39(-3.20,9.99)$

Abbreviations: BP, blood pressure; DBP, diastolic blood pressure; NA, not applicable; NHANES, National Health and Nutrition Examination Survey; SBP, systolic blood pressure.

NA means estimates cannot be obtained for the reason described in the note of Table 2.

Notes: data were adjusted for age, gender, education, family poverty income ratio, smoking, body mass index, serum cholesterol, use of medications for hypertension and diabetes.

${ }^{\ddagger} P<0.001,{ }^{*} P<0.05$ for the relative risks of uncontrolled BP, SBP and DBP and the differences in effects on continuous SBP and DBP for microalbuminuria and macroalbuminuria between racial groups.

The results were consistent with the findings from a retrospective longitudinal study in the Translational Research and Clinical Epidemiology (TRACE) Program. ${ }^{8}$ In the longitudinal study of resistant hypertensive patients in the TRACE program, researchers focused on the cohort of the majority of African Americans and investigated the effects of microalbuminuria and macroalbuminuria at the baseline on the later response of continuous SBP and DBP to antihypertensive treatment among African American hypertensive patients. They did not examine the relationships among microalbuminuria, macroalbuminuria and BP response in other racial groups because of the data limitations. However, in this study, we presented the associations of microalbuminuria and macroalbuminuria with uncontrolled $\mathrm{BP}$, isolated uncontrolled SBP and $\mathrm{DBP}$, as well as continuous SBP and DBP among non-Hispanic blacks, Mexicans and non-Hispanic whites. Uncontrolled BP may cause aneurysm, coronary heart disease, kidney failure and other life-threatening complications, ${ }^{18,19}$ and high SBP is a major health threat, especially for older Americans. ${ }^{19,20}$ The findings of the significant effects of albuminuria on later uncontrolled BP from the previous longitudinal cohort study, ${ }^{8}$ together with our results on the associations of albuminuria with uncontrolled BP and continuous SBP, imply that evaluating the albumin excretion rate regularly may be one strategy for improving the rate of hypertension control and reducing the risk of negative cardiovascular and renal outcomes for adults with diagnosed hypertension regardless of race.

After adjustment for potential confounding factors, the associations of microalbuminuria with uncontrolled BP and isolated uncontrolled SBP were significantly lower in non-Hispanic blacks and Mexicans than in non-Hispanic whites (Table 3). From the clinical point of view, the lower association of microalbuminuria with uncontrolled $\mathrm{BP}$ and isolated uncontrolled SBP in non-Hispanic blacks than in
non-Hispanic whites may be attributed to the more aggressive treatment of hypertension and its comorbidities in non-Hispanic black hypertensive subjects. In this study, although the rates of currently taking medications for hypertension are similar in nonHispanic blacks and whites (blacks $95.18 \%$ vs. whites $94.61 \%$ ), the rates of taking insulin for diabetes and taking medications for dyslipidemia were significantly higher in non-Hispanic blacks than in non-Hispanic whites $(9.43 \%$ vs. 5.13 for diabetes and $86.58 \%$ vs. $83.51 \%$ for dyslipidemia, both $P<0.05$ ).

The more aggressive treatment of comorbidities related to hypertension could be of benefit in lowering the levels of BP and weaken the association between microalbuminuria and both uncontrolled $\mathrm{BP}$ and isolated uncontrolled SBP among subjects with diagnosed hypertension in non-Hispanic blacks. The differences between Mexicans and non-Hispanic whites in the associations of microalbuminuria with uncontrolled BP and isolated uncontrolled SBP may be explained by the observation that the significantly higher prevalence of microalbuminuria in Mexicans than in non-Hispanic whites may sensitize health-care providers to its detection and treatment, which is evidenced by the finding that the average level of UACR (represented by the median) in Mexicans with microalbuminuria was significantly lower than in non-Hispanic whites with microalbuminuria $(51.8 \mathrm{vs}$. $\left.62.5 \mathrm{mg} \mathrm{g}^{-1}, P<0.05\right)$ in the present study. Although the prevalence of microalbuminuria was higher in Mexicans than in non-Hispanic whites, lower average levels of UACR could contribute to lower associations of microalbuminuria with uncontrolled BP and isolated uncontrolled SBP in Mexican hypertensive subjects.

Racial differences in the associations of macroalbuminuria with uncontrolled BP and other BP outcomes were not observed significantly among non-Hispanic blacks, Mexicans and non-Hispanic whites. The fact that macroalbuminuria is a more severe medical 
problem than microalbuminuria and more medical care may be given to people with macroalbuminuria may level out differences in the associations of macroalbuminuria with uncontrolled hypertension among racial groups. This is partly supported by the evidence from the data that the overall difference in median values of UACR is not significant among the three racial groups of hypertensive subjects with macroalbuminuria (results not shown). However, the higher prevalence of macroalbuminuria (non-Hispanic blacks $7.42 \%$ vs non-Hispanic whites $3.59 \%$ ) and the strong association of macroalbuminuria with all adverse $\mathrm{BP}$ outcomes (all $\mathrm{P}<0.05$ ) in non-Hispanic blacks with diagnosed hypertension (Table 2) indicate that non-Hispanic black hypertensive subjects still need more special care for severe albumin excretion problems to reduce the risk of adverse BP outcomes and cardiovascular disease-related mortality and morbidity in this population.

Hypertension control continues to be inadequate despite the array of effective and well-tolerated medications, and the low control rates can be attributed to poor management of elevated SBP. ${ }^{21}$ Moreover, high SBP has been thought to be a greater risk factor than DBP for the brain, heart, kidney and circulatory complications and for death, particularly in middle-aged and elderly adults. ${ }^{20,22-24}$ The findings in our study revealed that microalbuminuria was more associated with adverse SBP outcomes (isolated uncontrolled SBP and continuous SBP) than adverse DBP outcomes (isolated uncontrolled DBP and continuous DBP). Support from health-care providers and health insurers to more aggressively treat microalbuminuria may be a good strategy to have SBP controlled to be less than $140 \mathrm{~mm} \mathrm{Hg}$ to improve the control rates of hypertension and reduce the risks of adverse outcomes associated with high SBP among individuals with diagnosed hypertension.

There were several limitations in this study. First, continuous NHANES was a cross-sectional survey without any follow-up information for participants. Although the findings in the study could reflect the association of albuminuria with uncontrolled BP and continuous BP, they could not be used for causal inference for the effects of albuminuria on hypertension control. Second, diabetes may be underreported in the study because we only considered subjects who self-reported to have diabetes or were currently taking medications for diabetes and did not consider those who had undiagnosed or suspected diabetes. Third, there was no detailed drug information for hypertension in the NHANES survey. Although we controlled for the confounding impact from the general use of medications to treat hypertension, we could not obtain detailed information for the duration, type and dose of the drugs used. These factors could influence our results in this study. Fourth, there might be other unrecognized confounding factors (for example, duration of hypertension and treatment, lifestyle) that explain racial differences in albuminuria and its associations with uncontrolled BP and continuous BP. Finally, as a previous study noted, ${ }^{25}$ our definition of hypertension excluded hypertensive persons with BP successfully controlled by physical activity, weight control and other nonpharmacological techniques.

Differences in the rates of uncontrolled hypertension and associations of cardiovascular risk factors with the control of hypertension among non-Hispanic blacks, Mexicans and non-Hispanic whites could be attributed to genetic, cultural and social factors. 26-29 Further analysis of these factors among different racial groups would be helpful to better understand the role they have in the differential associations of cardiovascular risk factors with hypertension control. In our study, we explored racial differences in the associations of microalbuminuria and macroalbuminuria with uncontrolled BP and continuous BP using the continuous NHANES national survey data 1999-2008. The subjects who were not aware of their hypertension were excluded from the study because they may not have taken any actions to control high BP and the related-risk factors. The continuous NHANES survey was a large, stratified, multistage national survey of non-institutionalized participants with a complex probability design. The large sample size warranted the analysis power to find unbiased results that can reflect the real world. By considering the stratum, non-response adjustment and sampling weights, the results in our study can be generalized and applied to the general non-institutionalized population with diagnosed hypertension in the US.

In summary, diagnosed hypertensive subjects with microalbuminuria and macroalbuminuria were more likely to have uncontrolled BP and higher levels of SBP in each single population of non-Hispanic blacks, Mexicans and non-Hispanic whites. Macroalbuminuria was significantly associated with all adverse BP outcomes in non-Hispanic black hypertensives. Although the prevalence of microalbuminuria was higher in non-Hispanic blacks and Mexicans than in non-Hispanic whites, its associations with uncontrolled BP and isolated uncontrolled SBP were lower in the two racial populations. This has meant that hypertensive, non-Hispanic black or Mexican patients may have been more likely to have been treated by medical practitioners for microalbuminuria and its comorbidities, thereby weakening its relation to BP outcomes; however, nonHispanic whites with hypertension have not received the same high level of care as their non-Hispanic black and Mexican peers in monitoring and treating albuminuria because of the widely recognized lower risk. Because non-Hispanic whites account for the majority of the hypertensive population in the US, health providers need to further improve the care for mildly elevated albumin excretion rates in non-Hispanic white hypertensive patients while maintaining the quality care for non-Hispanic blacks and Mexicans to significantly lower the rates of uncontrolled hypertension and reduce the risk of adverse cardiovascular and renal outcomes in patients with diagnosed hypertension.

\section{CONFLICT OF INTEREST}

The authors declare no conflict of interest.

\section{ACKNOWLEDGEMENTS}

Research for this study was supported by an HRSA grant. We thank the National Center of Health Statistics for the availability of the NHANES survey data.

1 Stamler J, Stamler R, Neaton JD. Blood pressure, systolic and diastolic, and cardiovascular risks. US population data. Arch Intern Med 1993; 153: 598-615.

2 Smith SC Jr, Blair SN, Bonow RO. AHA/ACC guidelines for preventing heart attack and death in patients with atherosclerotic cardiovascular disease: 2001 update: a statement for healthcare professionals from the American Heart Association and the American College of Cardiology. J Am Coll Cardiol 2001; 38: 1581-1583.

3 Diringer MN. Management of aneurysmal subarachnoid hemorrhage. Crit Care Med 2009; 37: 432-440.

4 Levey AS, Coresh J, Balk E, Kausz AT, Levin A, Steffes MW, Hogg RJ, Perrone RD, Lau J, Eknoyan G. National Kidney Foundation Practice Guidelines for Chronic Kidney Disease: Evaluation, Classification, and Stratification. Ann Intern Med 2003; 139: 137-147.

5 Kannel WB. Blood pressure as a cardiovascular risk factor: prevention and treatment. JAMA 1996; 275: 1571-1576.

6 Kidney Disease Outcomes Quality Initiative (K/DOQI). K/DOQI clinical practice guidelines on hypertension and antihypertensive agents in chronic kidney disease. $\mathrm{Am} \mathrm{J}$ Kidney Dis 2004; 43 (5 Suppl 1), S1-290. 
7 Plantinga LC, Miller ER III, Stevens LA, Saran R, Messer K, Flowers N, Geiss L, Powe NR. Blood pressure control among persons without and with chronic kidney disease: US trends and risk factors 1999. Hypertension 2009; 54: 47-56.

8 Duncan K, Ohmit SE, Quah R, Liu X, Ramappa P, Norris S, Hedquist L, Dudley A, Nasser SA, Flack JM. Influence of albuminuria and glomerular filtration rate on blood pressure response to antihypertensive drug therapy. J Vasc Health Risk Manag 2007; 3: 1029-1037.

9 Ong KL, Tso AW, Lam KS, Cheung BM. Gender difference in blood pressure control and cardiovascular risk factors in Americans with diagnosed hypertension. Hypertension 2008; 51: 1142-1148.

10 Liu X, Liu M, Tsilimingras D, Schiffrin E. Racial disparities in cardiovascular risk factors among diagnosed hypertensive subjects. J Am Soc Hypertens 2011; 5: 239-248.

11 Young BA, Katon WJ, Von Korff M, Simon GE, Lin EH, Ciechanowski PS, Bush T, Oliver $M$, Ludman EJ, Boyko EJ. Racial and ethnic differences in microalbuminuria prevalence in a diabetes population: The Pathways Study. J Am Soc Nephrol 2005; 16: 219-228.

12 Egan BM, Zhao Y, Axon RN. US trends in prevalence, awareness, treatment, and control of hypertension, 1988-2008. JAMA 2010; 303: 2043-2050.

13 Ogunniyi MO, Croft JB, Greenlund KJ, Giles WH, Mensah GA. Racial/ethnic differences in microalbuminuria among adults with prehypertension and hypertension: National Health and Nutrition Examination Survey (NHANES), 1999-2006. Am J Hypertens 2010; 23: 859-864.

14 Chobanian AV, Bakris GL, Black HR, Cushman WC, Green LA, Izzo JL Jr, Jones DW, Materson BJ, Oparil S, Wright JT Jr, Roccella EJand the National High Blood Pressure Education Program Coordinating Committee. Seventh report of the Joint National Committee on Prevention, Detection, Evaluation, and Treatment of High Blood Pressure. Hypertension 2003; 42: 1206-1252.

15 Ezzati TM, Massey JT, Waksberg J, Chu A, Maurer KR. Sample design: Third Nationa Health and Nutrition Examination Survey. Vital Health Stat2 1992; 113: 1-35.

16 National Health and Nutrition Examination Survey. Physician examination procedures manual (January 2007) http://www.cdc.gov/nchs/data/nhanes/nhanes_07_08/manual_pe.pdf Accessed April 5, 2010.

17 Eknoyan G, Hostetter T, Bakris GL, Hebert L, Levey AS, Parving HH, Steffes MW, Toto R. Proteinuria and other markers of chronic kidney disease: a position statement of the national kidney foundation (NKF) and the national institute of diabetes and digestive and kidney diseases (NIDDK). Am J Kidney Dis 2003; 42: 617-622.

18 High blood pressure complications illustration. American Heart Association http:// www.americanheart.org/presenter.jhtml? identifier=3057206Accessed Nov. 9, 2010

19 High blood pressure. National Heart, Lung, and Blood Institute http://www.nhlbi.nih. gov/health/dci/Diseases/Hbp/HBP All.html Accessed Nov. 9, 2010.

20 Chobanian AV. Isolated systolic hypertension in the elderly. N Engl J Med 2007; 357 789-796.

21 Chobanian AV. The hypertension paradox-more uncontrolled disease despite improved therapy. N Engl J Med 2009; 361: 878-887.

22 Pini R, Cavallini MC, Bencini F, Silvestrini G, Tonon E, De Alfieri W, Marchionni N, Di Bari M, Devereux RB, Masotti G, Roman MJ. Cardiovascular remodeling is greater in isolated systolic hypertension than in diastolic hypertension in older adults: the Insufficienza Cardiaca negli Anziani Residenti (ICARE) a Dicomano Study. J Am Coll Cardiol 2002; 40: 1283-1289.

23 Ovbiagele B, Diener HC, Yusuf S, Martin RH, Cotton D, Vinisko R. Level of systolic blood pressure within the normal range and risk of recurrent stroke. Stroke $2011 ; 306$ : 2137-2144.

24 Cirillo M, Stellato D, Laurenzi M, Panarelli W, Zanchetti A, De Santo NG. Pulse pressure and isolated systolic hypertension: association with microalbuminuria. Kidney Int 2000; 58: 1211-1218.

25 Redmond N, Baer HJ, Hicks LS. Health behaviors and racial disparity in blood pressure control in the national health and nutrition examination survey. Hypertension 2011 , 57: 383-389.

26 Cholesterol Statistics. American Heart Association. 2008. Available at http://www .americanheart.org/presenter.jhtml?identifier=536 Accessed Sep 10, 2009.

27 Gerhard GT, Malinow MR, DeLoughery TG, Evans AJ, Sexton G, Connor SL, Wander RC, Connor WE. Higher total homocysteine concentrations and lower folate concentrations in premenopausal black women than in premenopausal white women. Am J Clin Nutr 1999; 70: 252-260.

28 Gao X, Bermudez OI, Tucker KL. Plasma c-reactive protein and homocysteine concentrations are related to frequent fruit and vegetable intake in hispanic and Non-Hispanic white elders. J Nutr 2004; 134: 913-918.

29 Celentano JC. Increased fruit and vegetable intake may reduce the nutrition-related health disparities in African Americans. Am J Lifestyle Med 2009; 3: 185-187. 